\title{
The Carl Sagan Observatory: A Telescope for Everyone
}

\author{
J. Saucedo-Morales, A. Sánchez-Ibarra
}

Area de Astronomia, DIFUS, Universidad de Sonora, Hermosillo, Sonora, México

D. Lunt

\section{Coronado Instruments Group}

\begin{abstract}
The Carl Sagan Observatory is a new project for a remote observatory that will be built at the summit of Cerro Azul (a $2480 \mathrm{~m}$ mountain located near Magdalena. Sonora, México). It will include one $55 \mathrm{~cm}$ and four $14 \mathrm{~cm}$ telescopes. The $55 \mathrm{~cm}$ telescope will be dedicated to supernovae research. One of the $14 \mathrm{~cm}$ Maksutov telescopes will be used as an autoguider for the stellar observations. The other $14 \mathrm{~cm}$ telescopes will feature different narrow band filters that will be used for solar research. The observatory will be controlled from the campus of the Universidad de Sonora in Hermosillo, Sonora, México ( $\sim 200 \mathrm{~km}$ from the site). A prototype of the observatory building has been built on campus and first light is expected by the end of May of 2001. We expect to have an operating mountain observatory by the end of 2002 . Some of the unique technical aspects of this observatory, which we believe can be a model for future small telescope observatories are discussed in this work.
\end{abstract}

\section{Introduction}

The Astronomy Area of the Center for Research in Physics, was established in 1990. It operates the only solar observatory in México, and a $41 \mathrm{~cm}$ stellar telescope, which is mostly used for educational purposes. The main research topics of the Area are: the activity of the solar corona and extragalactic astronomy. In 1996 a project for a remotely controlled solar-stellar telescope to be built at "Cerro Azul" was presented by the Astronomy Area to the Universidad de Sonora. After considering different options, it was decided in 1998 to commend the design and construction of the telescopes and optical instruments for the Carl Sagan Observatory Project (CSOP) to David Lunt (Coronado Instruments Group). The main features of the CSOP can be summarized as follows:

- A very good astronomical site

- Full use of technological innovations

- Low cost of construction and operation

- Outstanding optics in telescopes and instruments

- Maximum use of observing time (day and night operation) 
- Maximum use of the data (The observations will immediately be put on the Internet). This means that people from all over the world will have the same priority for the data use as the people on the staff from the CSOP.

- Aside from the scientific aspects of the project, a strong impact on educational and outreach programs is expected. It is for this reason that the new observatory will be named in memory of the great American astronomer and science educator Carl Sagan.

This paper concentrates on a discussion of the technical aspects of the telescopes, instruments and logistics of the CSOP. The scientific and educational objectives of the project, as well as additional information about the CSOP, can be found in Sánchez-Ibarra \& Saucedo-Morales (2001), as well as on the WEB page of the Astronomy Area (http://www.cosmos.astro.uson.mx). In sections 2 and 3 we discuss the different telescopes and instruments that will be available at the Carl Sagan Observatory. The logistical problems associated with a remote observatory (communications, electricity, control and maintenance) are briefly discussed in section 4 . The conclusions are given in section 5 .

\section{The Stellar Telescope}

The aperture of this telescope is $0.55 \mathrm{~m}$ and the focal length, $4.4 \mathrm{~m}$. The basis of the design is a folded, F/4 paraboloid with the image primary transferred by an ancillary optic lens group to a point behind the primary mirror which simultaneously doubles the focal length to the required $4.4 \mathrm{~m}$. Both the primary mirror and the flat are made of Zerodur. The primary has a thickness of $0.55 \mathrm{~mm}$ and this, combined with an open frame support system will allow the mirror to rapidly come to thermal equilibrium. The ancillary, transfer lens group includes a field lens and aperture stop to effectively eliminate spurious light from reaching the image plane. In order to keep the moments of inertia of the telescope to a minimum, the main tube consists of an octagonal section completely situated between the tines of the fork mount. The secondary mirror is supported on eight small diameter carbon fiber rods from the main box section resulting in a total mass of the secondary assembly of only $7 \mathrm{kgs}$. The primary mirror is mounted centrally within the box section on an open design support cell. The box section has 'shutter' sections to prevent sunlight from reaching the primary mirror when the instrument is used in solar mode. The mount is an equatorial fork. The main right ascension bearing is a 'Kaydon' precision turntable bearing. This provides four point support for the balls in the bearing. The force and moment specifications of the bearing are approximately 10 times the loads to be encountered in this instrument. The azimuth drive is a 1.75 inch precision worm wheel. The drive is a $30 \mathrm{VDC}$ stepping motor. The declination bearings are also 'Kaydon'. Both are 6-inch in diameter; one is of the same four point construction as the AZ bearing, the other is an axial support bearing.

The main instrument to be used in the stellar telescope will be a direct CCD camera, which will be used to image about 200 galaxies to search for supernovae every night. The CCD will be a high QE Apogee AP8 with a $1024 \times 1024$ format and a pixel size of $24 \mu \mathrm{m}$. With these characteristics, the stellar telescope will have a total field view of $\sim 19^{\prime} \times 19^{\prime}$, and a scale of $1.1^{\prime \prime} \mathrm{pix}^{-1}$ on the sky. The second instrument of the stellar telescope will be a spectrograph, whose main 
purpose will be the classification of supernovae. The rear end of the telescope will allow for rapid interchange from direct imaging to spectrograph mode by rotating a mirror.

\section{The Solar Telescopes}

The solar modules will rest on alternate sections of the octagonal box. This means that all the telescopes will share the same mount, which will greatly simplify the control of the telescopes. One of the most remarkable aspects of these modules is the surface smoothness of their optical components, which will be kept below $0.05 \mathrm{~nm}$ RMS to minimize the scatter from spurious sunlight. The first CCD detector to be installed in the solar modules is an Apogee Instruments AP10, which consists of a $2048 \times 2048$ Thompson CCD. One of the main reasons for choosing this camera for the solar telescope is its fast read out time which will make possible to study fast changes in the surface of the Sun. The plate scale for the solar modules will be $105^{\prime \prime} \mathrm{mm}^{-1}$, with a pixel size of $14 \mu \mathrm{m}$, with which the field of view will be large enough to cover the full disk of the Sun. Other instruments, such as a Kodak Megaplus camera are also expected to be installed at the telescope in the near future. This camera will make possible the transmission of the solar images in almost real time.

\section{The Site and Logistical Aspects of the CSOP}

Cerro Azul is located at West longitude 110 34', North latitude $3044^{\prime}$, with an elevation of $2480 \mathrm{~m}$ above sea level, in the Sonora-Arizona desert. It is $36 \mathrm{~km}$ from Magdalena, and close to $200 \mathrm{~km}$ from Hermosillo. It is a fairly isolated peak that it is expected to have a seeing of $\sim 1^{\prime \prime}$ (because the prevailing winds are expected to arrive at the observatory with close to laminar flows). The low level of light contamination at Cerro Azul was one of the reasons for choosing it as the site for the CSOP. There is however, a price one has to pay for this, since there are no electric power lines nor any other kind of basic services. Electric power will be obtained from solar energy. A network of solar cell panels and batteries will be installed outside the observatory building. The total power consumption is expected to be less than $2 \mathrm{KW}$. A back-up diesel generator, to be used in emergency situations, will also be available at the site.

Communications from Cerro Azul to the mountain called "Cerro de la Madera" (located $\sim 20 \mathrm{~km}$ to the west of Cerro Azul) will be through radiomodem transmission. From "La Madera" to Hermosillo the communication will be through optical fibers (that are property of the Mexican telephone company TELMEX). The transmission bandwidth will be about 256 KBPS at the beginning of the life of the observatory, but this is expected to increase by the time all the instruments are in full operation. The CSOP will also have a weather station, which will permit the computer at the site to close the dome and turn off the equipment and any instruments that are likely to be affected by bad weather. The weather station will play a more important role whenever the communication between Hermosillo and the observatory fails.

The Carl Sagan Observatory will be completely robotic and will be controlled from the University of Sonora in Hermosillo. We are considering several 
options for the software that will be used to control the observatory. At the moment, we are more inclined to use the Observatory Control and Astronomical Analysis System (OCAAS), sold by Torus Technologies. There will be a maintenance and alternative control station in Magdalena de Kino, Sonora. Service visits to the observatory site are expected to occur about every other week. No technical personnel are expected to be continuously present at the mountain once the CSOP is fully operational. This will permit to significantly reduce the cost of operation of the observatory. Due to the complexity of the problems related to the control of a mountain remote observatory, the telescopes, instruments, as well as all the equipment and materials that are going to be on the mountain, are first being thoroughly tested at the Universidad de Sonora. The pier, the prototype building (a cylindrical 3-D structure), and the dome (bought from Astrohaven) were built on campus to the same specifications as the ones that will be built on the mountain.

\section{Conclusions}

The CSOP is a big challenge, undertaken by a small university from a third world country interested in contributing to the overall knowledge of Astronomy. Aside from the scientific research that can be done with small telescope projects like the CSOP, these types of telescopes can be very powerful in increasing the interest in science. We also think that sharing information can be very profitable to both observatories and individuals interested in Astronomy. The total cost of the project will be around $\$ 250,000$ dollars, an extremely low cost compared with those of similar projects elsewhere. Taking into account the considerable return that can be obtained from a project like this, it should be expected that the number of remote observatories with small telescopes will grow in the near future.

Acknowledgments. The CSOP would not have been possible without the collaboration of several individuals. Here is a list of some of the people that have made the most substantial collaborations to the Project up to this time: José Martines Rocha, Carlos Méndez-Peón, Benito Noriega, José Fahra, Fernando Avila Castro, Fernando Diaz and Fernando Félix. We also like to acknowledge the continuous financial support from the Universidad de Sonora.

\section{References}

Sánchez-Ibarra, A. \& Saucedo-Morales, J. 2001, in ASP Conf. Ser. Vol. 225 eds. Robert J. Brunner, S. George Djorgovski, and Alex S. Szalay, in Press 